\title{
Auf Nummer sicher bei Garnelenallergie
}

\author{
Da allergische Reaktionen auf Krustentiere oft heftig sind und fatal \\ ausgehen können, ist die Diagnosesicherung besonders wichtig. Die \\ Ergebnisse von Anamnese, Pricktests und In-vitro-Diagnostik sind \\ jedoch nicht immer konsistent. Hier ist ergänzende Analytik gefragt.
}

rasilianische Forscher prüften, welchen Beitrag der Nachweis von spezifischen IgE-Antikörpern gegen Tropomyosin, das Hauptallergen von Garnelen, zur diagnostischen Sicherheit bei Garnelenallergie leisten kann. 35 Patienten mit Asthma, allergischer Rhinitis

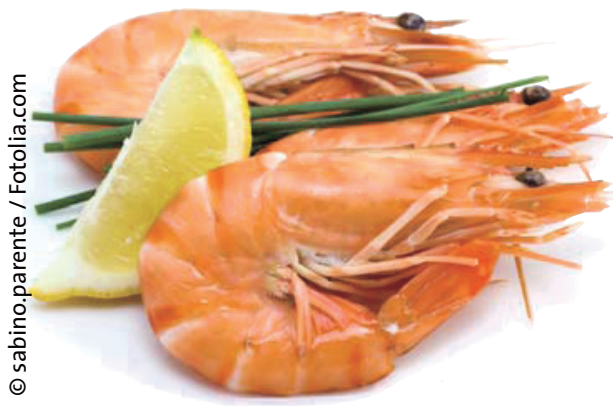

Bei Garnelenallergie kann der Nachweis von IgE-Antikörpern gegen Tropomyosin zur Diagnosesicherung beitragen. oder beidem, die gegen Dermatophagoides pteronyssinus sensibilisiert waren - das Tropomyosin von Garnelen weist einen hohen Grad an Homologie zu dem von Milben auf -, wurden zu Nahrungsmittelallergien und ihrem Konsum von Garnelenfleisch befragt. Zudem nahmen sie an einem Pricktest und an einem doppelblinden plazebokontrollierten oder auch offenen Provokationstest mit Garnelenfleisch teil. Die Antikörper wurden dann mittels CAP und chimärem ELISA bestimmt.

In den Provokationstests reagierten sieben Patienten auf Garnelen. Bei fünf von ihnen war auch das Ergebnis des In-vitro-Tests auf spezifische Antikörper gegen Tropomyosin positiv. Von den übrigen 28 Patienten zeigten im Invitro-Test nur zwei eine IgE-Antwort auf Tropomyosin, auf einen herkömm- lichen Garnelenextrakt dagegen sieben. Zehn der im Provokationstest nicht betroffenen Patienten reagierten im Pricktest positiv auf den Extrakt. Lag die Sensitivität für alle drei Methoden Pricktest, IgE-Antwort auf Garnelenextrakt und IgE-Antwort auf GarnelenTropomyosin - gleich hoch $(71,4 \%)$, unterschied sich die Spezifität deutlich. Sie lag bei $92,8 \%$ für die IgE-Antwort auf Tropomyosin, bei 75,0\% für die IgE-Antwort auf Garnelen und bei 64,2\% für die Reaktion im Pricktest.

Fazit: Die Bestimmung der spezifischen IgE-Antikörper gegen das Tropomyosin von Garnelen kann helfen, die Diagnose einer Garnelenallergie zu sichern. Die Verwendung des Hauptallergens statt des Gesamtextrakts erhöht die Spezifität der In-vitro-Diagnostik, ohne dass die Sensitivität leidet.

Yang AC et al. Measurement of IgE antibodies to shrimp tropomyosin is superior to skin prick testing with commercial extract and measurement of IgE to shrimp for predicting clinically relevant allergic reactions after shrimp ingestion. J Allergy Clin Immunol 2010; 125: 872-8

\section{Beikost: lieber früh als spät}

Jahrelang wurde empfohlen, potente Nahrungsmittelallergene möglichst spät in die Säuglingsernährung aufzunehmen. Dies hat sich in den letzten Jahren drastisch gewandelt, was sich auch in den aktuellen Leitlinien zur Allergieprävention widerspiegelt. Eine neue finnische Untersuchung unterstützt diesen Ansatz.

U rsache und Wirkung lassen sich in Studien zur Prävention von Nahrungsmittelallergien gerade bei familiär vorbelasteten Kindern oft nicht klar herausarbeiten. In einer neuen Untersuchung wurde deshalb auf die Daten einer prospektiven finnischen Geburtskohortenstudie mit 994 Kindern mit einer HLA-Risikokonstellation für Diabetes Typ I zurückgegriffen. Diese Studie lieferte Daten zur Stillzeit und der Einführung fester Nahrung. Fünfjährig wurden die Studienkinder dann von Allergologen auf Sensibilisierungen ge- gen häufige Allergene getestet, wie beispielsweise Ei, Kuhmilch, Fisch, Milbe, Katze, Birke und Gräser.

Ausschließlich gestillt wurde median über 1,8 Monate (0 bis 10 Monate). Nach Ausschluss von Störfaktoren waren folgende Zufütterungsmuster signifikant mit einer Sensibilisierung gegen Nahrungsmittelallergene assoziiert: späte Einführung von Kartoffeln (> 4 Monate), von Hafer, Roggen und Weizen (> 5 bzw. 7 bzw. 6 Monate), von Fleisch (> 5,5 Monate), von Fisch (> 8,2 Monate) und von Eiern (> 19,5 Monate).
Eine Assoziation mit der Sensibilisierung gegen Aeroallergene bestand bei der späten Einführung von Kartoffeln, Roggen, Fleisch und Fisch. Das höchste Risiko für eine Sensibilisierung gegen Nahrungsmittelallergene bestand bei später Zufütterung von Eiern, Hafer und Roggen, das höchste Risiko für eine Sensibilisierung gegen Aeroallergen bei später Zufütterung von Kartoffeln und Fisch. Die HLA-basierte Selektion der Kinder in dieser Studie dürfte keinen Einfluss auf die Ergebnisse genommen haben.

Fazit: Die verzögerte Einführung von bestimmten Beikostformen erhöht das Risiko für eine spätere Sensibilisierung gegen Aero- und Nahrungsmittelallergene im Alter von fünf Jahren. $\quad b k$

Nwaru Bl et al. Age at the introduction of solid foods during the first year and allergic sensitization at age 5 years. Pediatrics 2010; 125: 50-9 